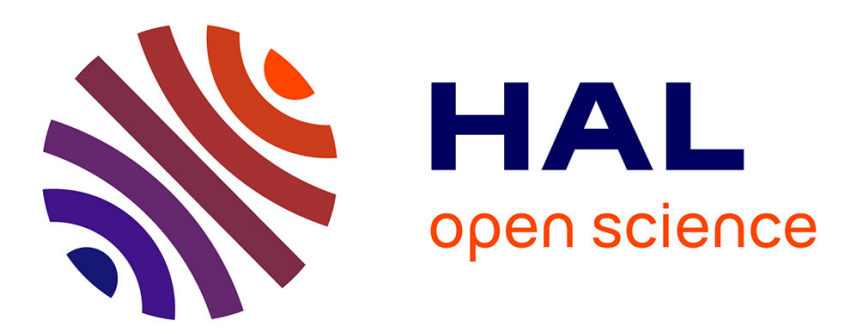

\title{
Fictitious Domains, Mixed Finite Elements and Perfectly Matched Layers for 2D Elastic Wave Propagation
}

\author{
Eliane Bécache, Patrick Joly, Chrysoula Tsogka
}

\section{To cite this version:}

Eliane Bécache, Patrick Joly, Chrysoula Tsogka. Fictitious Domains, Mixed Finite Elements and Perfectly Matched Layers for 2D Elastic Wave Propagation. [Research Report] RR-3889, INRIA. 2000. inria-00072764

\section{HAL Id: inria-00072764 \\ https://hal.inria.fr/inria-00072764}

Submitted on 24 May 2006

HAL is a multi-disciplinary open access archive for the deposit and dissemination of scientific research documents, whether they are published or not. The documents may come from teaching and research institutions in France or abroad, or from public or private research centers.
L'archive ouverte pluridisciplinaire HAL, est destinée au dépôt et à la diffusion de documents scientifiques de niveau recherche, publiés ou non, émanant des établissements d'enseignement et de recherche français ou étrangers, des laboratoires publics ou privés. 
INSTITUT NATIONAL DE RECHERCHE EN INFORMATIQUE ET EN AUTOMATIQUE

Fictitious domains, mixed finite elements and perfectly matched layers for $2 D$ elastic wave propagation

\author{
Eliane BECACHE, Patrick JOLY, Chrysoula TSOGKA
}

$\mathbf{N}^{\circ} 3889$

Mars 2000

THÈME 4 



\title{
Fictitious domains, mixed finite elements and perfectly matched layers for $2 \mathrm{D}$ elastic wave propagation
}

\author{
Eliane BECACHE* ${ }^{*}$ Patrick JOLY ${ }^{\dagger}$, Chrysoula TSOGKA \\ Thème 4 - Simulation et optimisation \\ de systèmes complexes \\ Projet Ondes \\ Rapport de recherche $\mathrm{n}^{\circ} 3889$ - Mars $2000-22$ pages
}

\begin{abstract}
We design a new and efficient numerical method for the modelization of elastic wave propagation in domains with complex topographies. The first specificity is the use of the fictitious domain method to take into account the boundary condition on the topography: the elastodynamic problem is extended in a domain with simple geometry, which permits the use of regular mesh. The free boundary condition is enforced introducing a Lagrange multiplier, defined on the boundary and discretized with a non uniform boundary mesh. This leads us to consider the first order velocity-stress formulation of the equations and particular mixed finite elements. These elements have three main non-standard properties: they take into account the symetry of the stress tensor, they are compatible with mass lumping techniques and lead to explicit time discretisation schemes, and they can be coupled with the Perfectly Matched Layer technique for the modeling of unbounded domains. Our method permits to model wave propagation in complex media such as anisotropic, heterogeneous media with complex topographies or/and with cracks, as it will be illustratred by several numerical experiments.
\end{abstract}

Key-words: mixed finite elements, mass lumping, anisotropic heterogeneous elastic waves, fictitious domain method, Perfectly Matched Layers

* INRIA-rocquencourt. Eliane.Becache@inria.fr

† INRIA-rocquencourt. Patrick.Joly@inria.fr

‡ INRIA-rocquencourt. Chrysoula.Tsogka@inria.fr

Unité de recherche INRIA Rocquencourt

Domaine de Voluceau, Rocquencourt, BP 105, 78153 LE CHESNAY Cedex (France)

Téléphone : 0139635511 - International : +33139635511

Télécopie : (33) 0139635330 - International : +33139635330 


\section{Domaines fictifs, éléments finis mixtes et couches absorbantes parfaitement adaptées pour la propagation des ondes élastiques 2D}

Résumé : Nous proposons une nouvelle méthode numérique performante pour la modélisation de la propagation d'ondes élastiques dans des domaines à topographies complexes. La première spécificité est l'utilisation de la méthode des domaines fictifs pour prendre en compte la condition aux limites sur la topographie: le problème élastodynamique est étendu à un domaine de géométrie simple, ce qui permet d'utiliser un maillage régulier. La condition aux limites de surface libre est imposée en introduisant un multiplicateur de Lagrange, défini sur la frontière et discrétisé sur un maillage non uniforme de la frontière. Ceci nous conduit à considérer la formulation des équations en système du premier ordre vitesse-contraintes et à utiliser des éléments finis mixtes particuliers. Ces éléments possèdent trois propriétés non standard : ils prennent en compte la symétrie du tenseur des contraintes, ils sont compatibles avec des techniques de condensation de masse qui conduisent à des schémas explicites, et ils peuvent être couplés avec la technique des couches absorbantes PML (Perfectly Matched Layers) pour la modélisation de domaines non bornés. Notre méthode nous permet de modéliser la propagation des ondes dans des milieux complexes, par exemple des milieux anistropes, hétérogènes à topographies complexes ou/et avec des fissures, comme cela sera illustré par plusieurs résultats numériques.

Mots-clé : éléments finis mixtes, condensation de masse, ondes élastiques anisotropes hétérogènes, méthode des domaines fictifs, couches absorbantes PML 


\section{Table of Contents}

1 Introduction $\quad 3$

2 The linear elastodynamic problem $\quad 5$

3 The Fictitious domain method for the elastodynamic problem 5

3.1 The mixed velocity-stress formulation $\ldots \ldots \ldots \ldots \ldots \ldots$

3.2 Application of the domain fictitious method to elastodymics . . . . . . . . . . . . . . 6

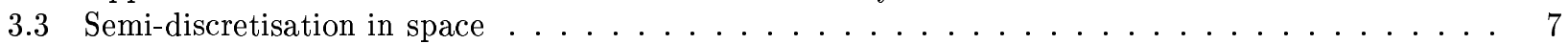

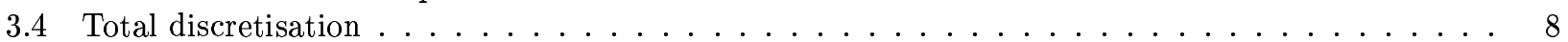

4 A new absorbing layer model (P.M.L) 9

4.1 The PML model for a general first order hyperbolic system . . . . . . . . . . . . . . 9

4.2 Application to elastodynamics . . . . . . . . . . . . . . . . . . . . 11

5 Construction of the $Q_{1}-Q_{0}$ mixed finite element $\quad 11$

6 Stability and dispersion analysis of the $Q_{1}-Q_{0}$ element in the case of a homogeneous, isotropic medium.

6.1 Interpretation as a finite difference scheme . . . . . . . . . . . . . . . . . . 14

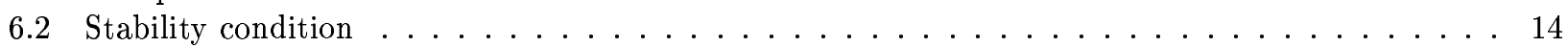

6.3 Dispersion Analysis for the total discretized scheme . . . . . . . . . . . . . . . . . . 16

7 Numerical Results $\quad 17$

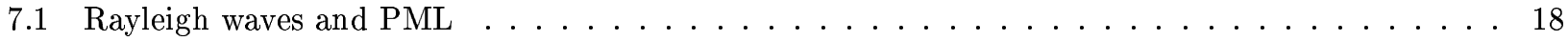

7.2 Homogeneous, anisotropic elastic medium. . . . . . . . . . . . . . . . . . . 19

7.3 Coupling with the fictitious domain method: the case of an heterogeneous elastic medium with complex topography . . . . . . . . . . . . . . . . 20

8 Conclusion

\section{Introduction}

In this work, we present a fictitious domain method for modeling time dependent elastic wave propagation in complex media such as heterogeneous anisotropic media of complex geometries. From an industrial point of view, the main applications concerned by this work are the study of seismic waves in media with complex topographies and the non destructive testing (diffraction by a crack of complex geometry). In this paper we will restrict ourseleves in the first application considering the elastodynamic problem in a domain with a complex topography (see Fig. 1).

Among the possible methods for solving this problem, the finite difference method is one of the most attractive. It uses a regular mesh with an explicit time discretisation, and therefore is very efficient from the computational point of view. Its main drawback is that it produces spurious diffractions when the boundary does not fit the grid mesh (see Fig 1-left). An alternative is to use finite elements with a nonuniform mesh,

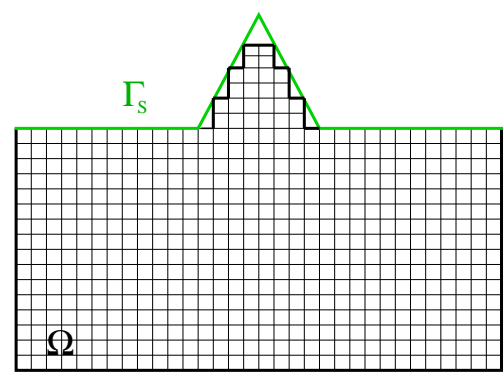

$\Gamma_{\mathrm{D}}$

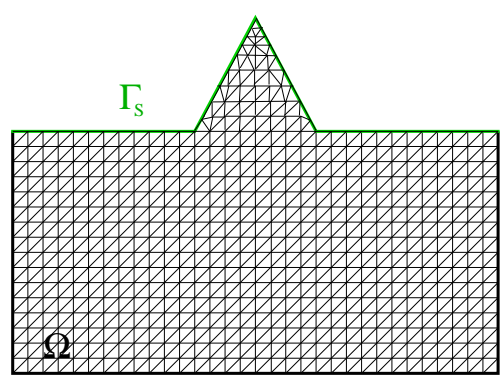

$\Gamma_{\mathrm{D}}$

Figure 1: Left: finite difference method (staircase approximation). Right: Finite elements 
that can fit exactly the complex geometry of the boundary, see Fig. 1-right. Nevertheless, other disadvantages are introduced. The numerical implementation is much more difficult, the efficiency may be decreased by the unstructured nature of the data, and finally the CFL stability is affected: in order to fit the complex geometry of the boundary, the mesh may contain elements of very small size, which implies, because of the CFL stability condition, the use of a very small time step if one uses an explicit scheme.

We propose here the use of an alternative method, the fictitious domain method (also called the domain embedding method) which combines the efficiency of the finite difference method (regular meshes and explicit time discretisation schemes) with a good approximation of the topography. This method was initially developed for solving problems with complex geometries[2, 16], particularly in the stationary case. The study of the fictitious domain method for time dependent problems has started few years ago[17, 12, 15]. Its principle is to extend the solution to a simple shape domain (typically a rectangle in 2D) containing the domain of interest, independent of the boundary of complex geometry, and to impose the boundary condition via the introduction of a Lagrange multiplier on the boundary. The main point is that we have now two unknowns, the extended function, defined in the enlarged simple shape domain and the auxiliary variable, defined on the boundary of complex geometry, so that the mesh for computing the extended function can be chosen independently of the geometry of the boundary. In particular, the use of regular grids allows simple and efficient computations. Of course, we have to pay for this advantage in terms of some additional computational cost due to the determination of the new boundary unknown. However, the final numerical scheme appears to be a slight perturbation of the scheme for the problem without obstacle so that this cost may be considered as marginal.

In the case of an elastic medium the boundary condition is a free surface condition, that means, the normal stress is zero on the surface : in order to consider this condition as an equality constraint, we are led in a natural way to use the mixed velocity-stress formulation for elastodynamics. Then the Lagrange multiplier can be interpreted as the jump of the velocity through the surface. Another advantage of working with the first-order mixed velocity-stress formulation is that it is well suited to the use of a new absorbing layer model for bounding the computational domain: the Perfectly Matched Layers (PML), introduced by Berenger[10] for the 2D Maxwell problem and that can be extended to elastodynamics. This model has astonishing properties : the reflection coefficient at the interface between the layer and the free medium is zero whatever are the frequency and the angle of incidence. The extension of this model to elastodynamics is natural when using the mixed velocity-stress formulation.

These considerations lead us to find an efficient approximation of the time domain mixed velocity-stress formulation. In particular for stability reasons (conservation of energy), we have decided to use a discretisation procedure in space based on a variational formulation of the velocity stress system, which is a first order hyperbolic system. At this stage, our main requirement is to define a spatial discretisation which allows the obtention of an explicit time discretisation scheme (mass-lumping).

Several mixed finite element methods are proposed in the literature especially for plane elasticity. We refer for example to PEER'S element introduced by D. N. Arnold, F. Brezzi and J. Douglas[1] and more recently to the work of R. Stenberg[20] and M. Morley[18]. One of the well known difficulties for mixed elements in elasticity is to take into account the symmetry of the stress tensor. The method used in these papers $[1,20,18]$ consists in working with a space of non necessarily symmetric tensors and imposing the symmetry in a weak way. Namely, the symmetry is enforced via the introduction of a Lagrange multiplier. Although these methods are very interesting for the plane elasticity problem, we did not retain them as they lead to an implicit scheme in time.

That is why we have constructed an original mixed finite element (inspired from the second Nedelec's family[19]) using spaces of symmetric tensors for the stress[7]. These spaces will fit our objectives.

The error analysis of these mixed finite elements will not be discussed here, we refer the reader to[9, 8], where a non classical convergence theory is presented.

The present paper is organized as follows. In $\S 2$ we briefly recall the equations of elastodynamics. In $\S 3$ we describe the fictitious domain method applied to elastodynamics, with a free surface condition on the boundary. We explain in $\S 4$ how to apply the PML to the elastodynamic problem. We introduce in $\S 5$ the new family of mixed finite elements for linear elasticity, which permits us to make mass lumping. The dispersion and stability analysis of the lowest order element is presented in $\S 6$ in the case of an homogeneous isotropic medium. Finally, we show in $\S 7$ some numerical results. 


\section{The linear elastodynamic problem}

We want to solve the linear elastodynamic problem with a complex topography (see Figure 2-left). The solution is governed by the elastic wave equation in $\Omega$ and we impose the free surface condition on $\Gamma_{S}$. For the sake of simplicity a Dirichlet condition is assumed on the exterior boundary $\Gamma_{D}$ but we will see in section 4 how we can take into account the modelisation of an unbounded domain by an efficient absorbing layer model (PML).

Notations. In the following, we identify the space of $2 \times 2$ tensors with the space $\mathcal{L}\left(\mathbb{R}^{2}\right)$ of linear applications from $\mathbb{R}^{2}$ to $\mathbb{R}^{2}$. We define the linear form, as $(\sigma)=\sigma_{12}-\sigma_{21}$, and the subspace of symmetric tensors of $\mathcal{L}\left(\mathbb{R}^{2}\right)$ :

$$
\mathcal{L}^{s}\left(\mathbb{R}^{2}\right)=\left\{\sigma \in \mathcal{L}\left(\mathbb{R}^{2}\right) / \mathbf{a s}(\sigma)=0\right\} .
$$

The scalar product in $\mathcal{L}\left(\mathbb{R}^{2}\right)$ is defined by $\sigma: \tau=\sigma_{i j} \tau_{i j}$, forall $(\sigma, \tau)$ in $\mathcal{L}\left(\mathbb{R}^{2}\right)$ and $|\sigma|$ is the associated norm. Finally, the divergence of a tensor is defined as:

$$
\operatorname{div} \sigma=\left[\begin{array}{c}
\frac{\partial \sigma_{11}}{\partial x_{1}}+\frac{\partial \sigma_{12}}{\partial x_{2}} \\
\frac{\partial \sigma_{21}}{\partial x_{1}}+\frac{\partial \sigma_{22}}{\partial x_{2}}
\end{array}\right] .
$$

The continuous displacement problem. We consider now the following elastodynamic problem :

$$
\begin{cases}\varrho \frac{\partial^{2} u}{\partial t^{2}}-\operatorname{div} \sigma(u)=g & \text { in } \Omega \\ u=0 & \text { on } \Gamma_{D} \\ \sigma \cdot n=0 & \text { on } \Gamma_{S}\end{cases}
$$

with some initial conditions at time $t=0$ that we will systematically omit in the following. In $(1), u=\left(u_{1}, u_{2}\right)^{t}$ denotes the displacement, $\sigma(u)$ is the stress tensor and $\varrho=\varrho(x)$ is the density. Consider $\varepsilon(u)$ the strain tensor, i.e.,

$$
\varepsilon_{i j}(u)=\frac{1}{2}\left(\frac{\partial u_{i}}{\partial x_{j}}+\frac{\partial u_{j}}{\partial x_{i}}\right)
$$

The stress tensor is related to the strain tensor by Hooke's law

$$
\sigma(u)(x, t)=C(x) \varepsilon(u)(x, t),
$$

where $C(x)$ is a $4 \times 4$ positive tensor having the classical properties of symmetry[3]. The density is assumed to be bounded,

$$
0<\varrho_{-} \leq \varrho(x) \leq \varrho_{+}<+\infty \quad \text { p.p. } x \in \Omega,
$$

In the following, we set $A(x)=C(x)^{-1}$ and we suppose that $A(x)$ satisfies :

$$
\forall \sigma \in \mathcal{L}^{s}\left(\mathbb{R}^{2}\right) \quad 0<\alpha|\sigma|^{2} \leq A(x) \sigma: \sigma \leq M|\sigma|^{2}
$$

\section{The Fictitious domain method for the elastodynamic problem}

In this section we apply the fictitious domain method for solving the elastodynamic problem (1). As explained in the introduction, this method consists in extending the solution of problem (1) to a larger domain of simple geometry (see Fig 2) and in taking into account the boundary condition in a weak way, thanks to the introduction of a Lagrange multiplier. To do so with the free surface condition (Neumann condition), $\sigma$ has necessarily to be one of the unknowns. Therefore, we have to write the elastodynamic problem (1) as a first order hyperbolic system, the so called velocity-stress system.

Remark 1 More generally, the fictitious domain method can be used for the essential conditions, i.e. conditions that can be taken into account in the functional space.

$\mathrm{RR} \mathrm{n}^{\circ} 3889$ 


\subsection{The mixed velocity-stress formulation}

Problem (1) is equivalent to the mixed velocity-stress system :

$$
\left\{\begin{array}{llll}
\varrho \frac{\partial v}{\partial t}-\operatorname{div} \sigma & = & \text { in } \Omega & (i) \\
A \frac{\partial \sigma}{\partial t}-\varepsilon(v) & = & 0 & \text { in } \Omega
\end{array}\right.
$$

where $v=\frac{\partial u}{\partial t}$ is the velocity in $\Omega$ and $f=\frac{\partial g}{\partial t}$. Consider the spaces

$$
\left\{\begin{array}{l}
X=\left\{\tau \in(H(\operatorname{div}, \Omega))^{2}, \tau \cdot \vec{n}=0 \text { on } \Gamma_{S}\right\}, \\
X^{s}=\{\tau \in X, \tau \text { symmetric }\}, \\
M=\left(L^{2}(\Omega)\right)^{2},
\end{array}\right.
$$

the variational formulation of problem $(2)$ is :

$$
\begin{cases}\text { Find }(\sigma, v) \in X^{s} \times M \text { such that: } & \\ \frac{d}{d t} a(\sigma, \tau)+b(\tau, v)=0, & \forall \tau \in X^{s}, \\ \frac{d}{d t} c(v, w)-b(\sigma, w)=(f, w), & \forall w \in M,\end{cases}
$$

where we have set :

$$
\begin{cases}a(\sigma, \tau)=\int_{\Omega} A \sigma: \tau d x, & \forall(\sigma, \tau) \in X^{s} \times X^{s}, \\ c(v, w)=\int_{\Omega} \varrho v \cdot w d x, & \forall(v, w) \in M \times M \\ b(\tau, w)=\int_{\Omega} \operatorname{div} \tau \cdot w d x, & \forall(\tau, w) \in X^{s} \times M .\end{cases}
$$
This system satisfies the classical energy estimate $\mathbf{E}(t)=\mathbf{E}(0)+\int_{0}^{t}(f(s), v(s))_{M} d s$, the energy being defined
as

$$
\mathbf{E}(t)=\frac{1}{2}(A \sigma, \sigma)_{H}+\frac{1}{2}(\rho v, v)_{M} \equiv \frac{1}{2} a(\sigma, \sigma)+\frac{1}{2} c(v, v)
$$

Remark 2 To obtain this formulation it is crucial to work in the space $X^{s}$ of symmetric tensors : only in this space operators $-\varepsilon$ and div are adjoint.

\subsection{Application of the domain fictitious method to elastodymics}

We now extend solution of problem (2) to the solution, still denoted $(v, \sigma)$ for simplicity, of a problem posed in an enlarged domain $C$, which is a simple rectangle (see Fig 2), with Dirichlet conditions on the external boundary,

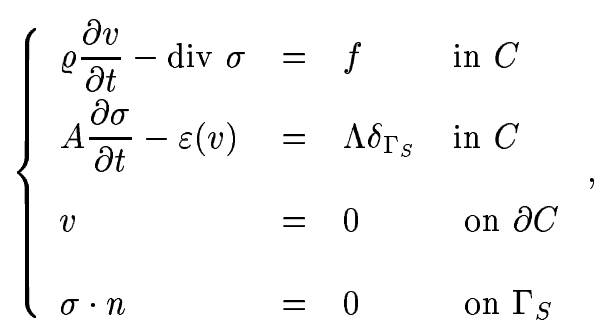


where $\Lambda$ is the tensor of components $\Lambda_{i j}=\lambda_{i} n_{j}, \lambda$ being a new unknown, only defined on the boundary $\Gamma_{S}$. We introduce the spaces (denoted also $X, X^{s}$ and $M$ for simplicity)

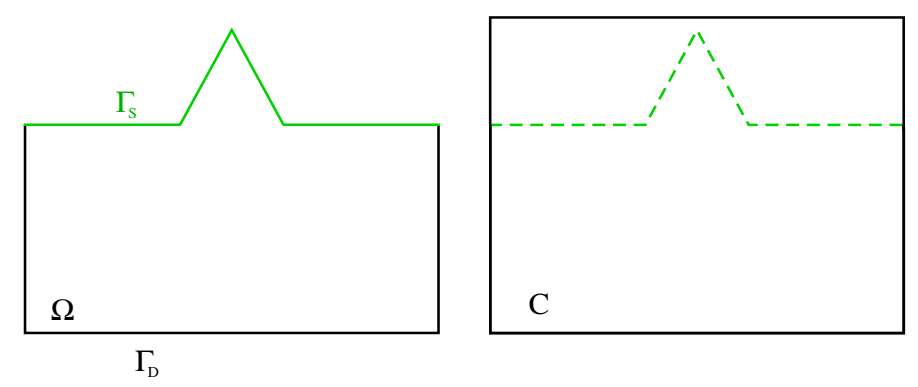

Figure 2: Left: the original domain. Right: the enlarged domain

$$
\left\{\begin{array}{l}
X=\left\{\tau \in(H(\operatorname{div}, C))^{2}\right\} ; X^{s}=\{\tau \in X, \tau \text { symmetric }\}, \\
M=\left(L^{2}(C)\right)^{2} ; \mathcal{G}=\left(H^{1 / 2}\left(\Gamma_{S}\right)\right)^{2},
\end{array}\right.
$$

defined on the whole rectangle $C$. The variational formulation of (5) can be written as follows:

$$
\begin{cases}\text { Find }(\sigma, v, \lambda) \in X^{s} \times M \times \mathcal{G} \text { such that: } & \\ \frac{d}{d t} a(\sigma, \tau)+b(\tau, v)-s(\tau, \lambda)=0, & \forall \tau \in X^{s} \quad(i) \\ \frac{d}{d t} c(v, w)-b(\sigma, w)=(f, w), & \forall w \in M \quad(i i) \\ s(\sigma, \mu)=0, & \forall \mu \in \mathcal{G} \quad(i i i)\end{cases}
$$

the bilinear forms $a(\cdot, \cdot), b(\cdot, \cdot), c(\cdot, \cdot)$ being defined by (4) (with $C$ instead of $\Omega$ ), with

$$
s(\tau, \lambda)=\langle\tau \vec{n}, \lambda\rangle_{\Gamma_{S}} \quad \forall(\tau, \lambda) \in X^{s} \times \mathcal{G} .
$$

Actually the unknown $\lambda$ can be interpreted as a Lagrange multiplier corresponding to $[v]_{\Gamma_{S}}$. Obviously, the restriction of $(v, \sigma)$ to $\Omega$ still satisfies (2). Moreover, we can remark that the restriction of the solution to $\bar{\Omega}^{c}$ (where $\bar{\Omega}^{c}$ denotes the complementary of $\bar{\Omega}$ in $C$ ) also satisfies (2) where $\Omega$ is replaced by $\bar{\Omega}^{c}$ (and $\Gamma_{D}$ denotes $\partial C \cap \partial \bar{\Omega}^{c}$ ). Now if we multiply (2)-(ii) with a function $\tau \in X^{s}\left(X^{s}\right.$ being defined by (6)), and integrate in $\Omega \cup \bar{\Omega}^{c}$, an integration by parts of the second term gives

$$
-\int_{\Omega \cup \Omega^{c}} \varepsilon(v) \tau d x=\int_{\Omega \cup \Omega^{c}} v \cdot \operatorname{div} \tau d x-\left\langle\tau \vec{n}, v^{+}-v^{-}\right\rangle_{\Gamma_{S}} \equiv b(\tau, v)-s\left(\tau,[v]_{\Gamma_{S}}\right),
$$

which yields (7-(i)), if we set $\lambda=[v]_{\Gamma_{S}}$. Since the free surface boundary condition on $\Gamma_{S}$ is not taken into account anymore in the new definition of space $X$, it has to be imposed in the formulation, this is done with (7-(iii)).

Remark 3 There is a strong link between the fictitious domain method and the boundary integral equation method. The extended solution is chosen such that the normal stress is continuous through $\Gamma_{S}$ and the new unknown corresponds to the unknown of the BIE obtained using a double layer potential representation for v[5].

\subsection{Semi-discretisation in space}

Consider now the finite dimension spaces $X_{h}^{s} \subset X^{s}, M_{h} \subset M$ and $\mathcal{G}_{h} \subset \mathcal{G}$ with the classical approximation properties:

$\mathrm{RR} \mathrm{n}^{\circ} 3889$

$$
\begin{aligned}
& \inf _{\tau_{h} \in X_{h}^{s}}\left\|\sigma-\tau_{h}\right\|_{X^{s}}=0, \quad \forall \sigma \in X^{s} \\
& \inf _{w_{h} \in M_{h}}\left\|v-w_{h}\right\|_{M}=0, \quad \forall v \in M
\end{aligned}
$$$$
\inf _{\mu_{h} \in \mathcal{G}_{h}}\left\|\lambda-\mu_{h}\right\|_{\mathcal{G}}=0, \quad \forall \lambda \in \mathcal{G}
$$ 
The semi discretisation in space of problem (7) can be written:

$$
\begin{cases}\text { Find }\left(\sigma_{h}, v_{h}, \lambda_{h}\right) \in X_{h}^{s} \times M_{h} \times \mathcal{G}_{h} \text { such that : } & \\ \frac{d}{d t} a\left(\sigma_{h}, \tau_{h}\right)+b\left(\tau_{h}, v_{h}\right)-s\left(\tau_{h}, \lambda_{h}\right)=0, & \forall \tau \in X_{h}^{s} \\ \frac{d}{d t}\left(v_{h}, w_{h}\right)-b\left(\sigma_{h}, w_{h}\right)=\left(f, w_{h}\right), & \forall w_{h} \in M_{h} \\ \left\langle\sigma_{h} \vec{n}, \mu_{h}\right\rangle_{\Gamma_{S}}=0, & \forall \mu_{h} \in \mathcal{G}_{h}\end{cases}
$$

We introduce here $B_{N_{1}}=\left\{\tau_{i}\right\}_{i=1}^{N_{1}}, B_{N_{2}}=\left\{w_{i}\right\}_{i=1}^{N_{2}}$ and $B_{N_{3}}=\left\{\mu_{i}\right\}_{i=1}^{N_{2}}$ the bases of $X_{h}^{s}, M_{h}$ and $\mathcal{G}_{h}$ respectively $\left(N_{1}=\operatorname{dim} X_{h}^{s}, N_{2}=\operatorname{dim} M_{h}\right.$ and $\left.N_{3}=\operatorname{dim} \mathcal{G}_{h}\right),\left[\Sigma_{h}\right]=\left(\Sigma_{1}, \ldots, \Sigma_{N_{1}}\right),\left[V_{h}\right]=\left(V_{1}, \ldots, V_{N_{2}}\right)$ and $\left[\Lambda_{h}\right]=$ $\left(\Lambda_{1}, \ldots, \Lambda_{N_{3}}\right)$ the coordinates of $\sigma_{h}, v_{h}$ and $\lambda_{h}$ in the bases $B_{N_{1}}, B_{N_{2}}$ and $B_{N_{3}}$. In these bases, (8) can be written in the following matricial form:

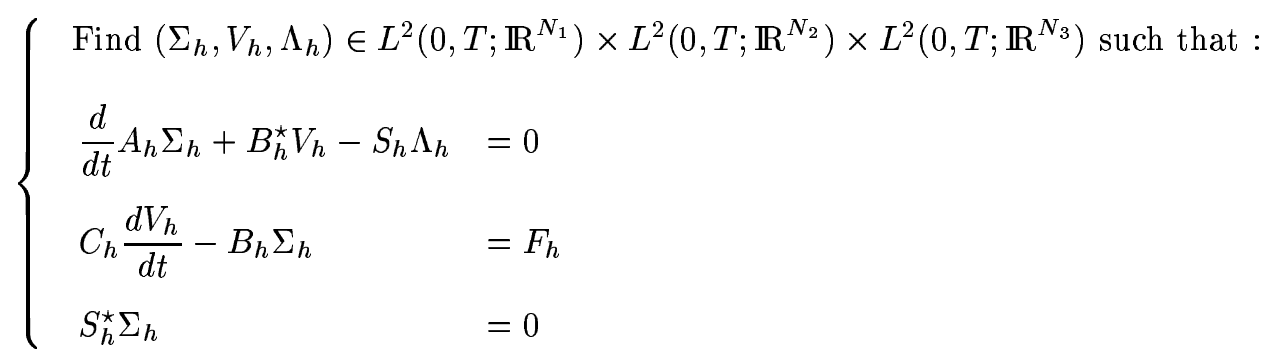

where $M^{\star}$ denotes the transpose of the matrix $M$. In practice, and this is the interesting point in the fictitious domain method, we introduce two meshes: the volumic unknowns $\left(V_{h}, \Sigma_{h}\right)$ are defined on a regular grid, while the surfacic unknown $\Lambda_{h}$ is computed on a nonuniform mesh on $\Gamma_{S}$, see Fig. 13-left. From the theoretical point of view, the well-posedness of problem (9) and the convergence of the method is linked to the obtention of a uniform inf-sup condition which leads to a compatibility condition between the boundary mesh and the uniform mesh[23]. Practically, if $H$ denotes the boundary mesh stepsize and $h$ the grid stepsize, it suffices that $H \geq 1.5 h$.

At this point we can see the importance of mass-lumping: assume for the moment that we can find appropriate finite element spaces and the adequate quadrature formulas in order to achieve mass-lumping on the matrix $A_{h}$, we can then eliminate the unknown $\Sigma_{h}$ (which implies important savings in memory requests especially in the $3 \mathrm{D}$ case) and write system (9) as the second order system in time :

$$
\begin{cases}\text { Find }\left(V_{h}, \Lambda_{h}\right) \in L^{2}\left(0, T ; \mathbb{R}^{N_{2}}\right) \times L^{2}\left(0, T ; \mathbb{R}^{N_{3}}\right) & \text { such that : } \\ C_{h} \frac{d^{2} V_{h}}{d t^{2}}+B_{h} A_{h}^{-1} B_{h}^{\star} V_{h}-B_{h} A_{h}^{-1} S_{h} \Lambda_{h} & =\frac{d F_{h}}{d t} \\ -S_{h}^{\star} A_{h}^{-1} B_{h}^{\star} V_{h}+S_{h}^{\star} A_{h}^{-1} S_{h} \Lambda_{h} & =0\end{cases}
$$

We will describe in section 5 an appropriate choice for $X_{h}^{s}, M_{h}$ (corresponding to the lowest order element of a new family of mixed finite elements) which allows to obtain mass-lumping for $A_{h}$.

\subsection{Total discretisation}

For the time discretisation of problem (10) we restrict ourselves to the classical second order centered finite differences approximation :

$$
\left\{\begin{aligned}
\text { Find }\left(V_{h}^{n+1}, \Lambda_{h}^{n}\right) \in \mathbb{R}^{N_{2}} \times \mathbb{R}^{N_{3}} \text { such that : } & \\
C_{h} \frac{V_{h}^{n+1}-2 V_{h}^{n}+V_{h}^{n-1}}{\Delta t^{2}}+B_{h} A_{h}^{-1} B_{h}^{\star} V_{h}^{n}-B_{h} A_{h}^{-1} S_{h} \Lambda_{h}^{n} & =\frac{F_{h}^{n+1 / 2}-F_{h}^{n-1 / 2}}{\Delta t} \\
S_{h}^{\star} A_{h}^{-1} S_{h} \Lambda_{h}^{n} & =S_{h}^{\star} A_{h}^{-1} B_{h}^{\star} V_{h}^{n}
\end{aligned}\right.
$$


This is an explicit scheme only if $C_{h}$ is also a diagonal matrix, which, in practice, is not difficult to achieve. This comes from the fact that $M_{h}$ is a subset of $L^{2}$ and therefore can be constructed with discontinuous fonctions and from now on we assume that $C_{h}$ is diagonal. The additional terms, coming from the coupling with the fictitious domain method are the terms containing the unknown $\Lambda_{h}^{n}$. This means that, without topography, we would have to solve

$$
\frac{V_{h}^{n+1}-2 V_{h}^{n}+V_{h}^{n-1}}{\Delta t^{2}}+C_{h}^{-1} B_{h} A_{h}^{-1} B_{h}^{\star} V_{h}^{n}=C_{h}^{-1} \frac{F_{h}^{n+1 / 2}-F_{h}^{n-1 / 2}}{\Delta t}
$$

which can be reinterpreted as a finite difference scheme (see section 6) and is comparable from the computational point of view to the classical finite difference scheme. Therefore, the additional cost for taking into account the topography thanks to the fictitious domain method, compared to the classical finite difference scheme, is due to the system (11)-(ii). The matrix of this system is of small size (number of degrees of fredom on $\Gamma_{S}$ ) and independent on the step $n$, so that it can be factorized once and we only have to perform a forward backward solve at each time step. Note that the term $\Lambda_{h}^{n}$ in equation (11)-(i) can be interpreted as an additional source term located on the boundary.

Remark 4 - System (10) has the advantage of being a second-order system in time : it is easier to get higherorder discretisation in time, using the modified equation technique (see[14]), than for the first-order system.

- The invertibility of the matrix $S_{h}^{\star} A_{h}^{-1} S_{h}$ of System (11)-(ii) (and thus the well posedness of ((11)) is insured by the inf-sup condition already mentioned before (needed for the convergence of the method).

An important point is that the CFL stability condition is the same for (11) than for (12), i.e. for the discretized problem posed in the whole rectangle $C$ without topography, which means that the use of the fictitious domain method does not affect the stability condition. Actually, assume that there is no source term (i.e., $F=0$ ), then we can prove that, for the solution of (11), the following quantity is conserved

$$
E^{n+1 / 2}=\left(C_{h} \frac{V_{h}^{n+1}-V_{h}^{n}}{\Delta t}, \frac{V_{h}^{n+1}-V_{h}^{n}}{\Delta t}\right)+\left(B_{h} A_{h}^{-1} B_{h}^{\star} V_{h}^{n+1}, V_{h}^{n}\right)
$$

which is exactly the discrete energy of (12) (see[12] for more details).

We will see in section 6.2 what is the stability condition for our particular choice of spaces $X_{h}^{s}$ and $M_{h}$, in a case of an homogeneous, isotropic elastic medium.

\section{A new absorbing layer model (P.M.L)}

A new absorbing layer model, the Perfectly Mached Layer model was introduced by Berenger[10] for the 2D Maxwell problem. This model has astonishing properties: the reflection coefficient at the interface between the layer and the free medium is zero whatever are the frequency and the angle of incidence. This model can be extended to general first order hyperbolic systems, and in particular to the first order velocity-stress formulation (see[13] for more details). In this section, we explain the basic principles of this model in the general case of a first order hyperbolic system and then we will extend this model to elastodynamics.

\subsection{The PML model for a general first order hyperbolic system}

Consider the following first order hyperbolic system, posed initially in the space $\mathbb{R}^{2}$ :

$$
\begin{cases}\frac{\partial u}{\partial t}=A \frac{\partial u}{\partial x_{1}}+B \frac{\partial u}{\partial x_{2}}, & u \in \mathbb{R}^{m} \\ u\left(x_{1}, x_{2}, 0\right)=u^{0}\left(x_{1}, x_{2}\right) & (a)\end{cases}
$$

Suppose that the support of initial data $u^{0}$ is in the left half-space, we would like to substitute problem (13) by an equivalent one posed in the left half-space. The basic principle of the PML model is to couple the equation in the left half-space with an equation in the right half-space such that there is no reflection at the interface 
and that the wave decreases exponentially inside the layer. We first introduce the following system

$$
\left\{\begin{array}{l}
u=u^{\|}+u^{\perp} \\
\frac{\partial u^{\|}}{\partial t}=B \frac{\partial u}{\partial x_{2}} \\
\frac{\partial u^{\perp}}{\partial t}=A \frac{\partial u}{\partial x_{1}}
\end{array}\right.
$$

where the index $\|$ (resp. $\perp$ ) means that we keep only the derivatives parallel to the interface, i.e. the $x_{2^{-}}$ derivatives (resp. orthogonal, i.e., the $x_{1}$-derivatives). Is is easy to see that system (14) implies (13)-(a).

Secondly we define a new wave, $v$, solution of (14) in the left half-space and satisfying a new system in the right half-space, involving a damping on the normal component :

$$
\left\{\begin{array}{l}
v=v^{\|}+v^{\perp} \\
\frac{\partial v^{\|}}{\partial t}=B \frac{\partial v}{\partial x_{2}} \\
\frac{\partial v^{\perp}}{\partial t}+d\left(x_{1}\right) v^{\perp}=A \frac{\partial v}{\partial x_{1}}
\end{array}\right.
$$

where the damping parameter $d\left(x_{1}\right)$ is positif and satisfies :

$$
d\left(x_{1}\right)=0, \quad \forall x_{1} \leq 0 .
$$

Now, consider a plane wave $u$, solution of (13)-(a), i.e., on the form :

$$
u\left(x_{1}, x_{2}, t\right)=u_{0} e^{-i\left(k_{1} x_{1}+k_{2} x_{2}-\omega t\right)},
$$

where $u_{0}$ satisfies the dispersion relation :

$$
u_{0}+\frac{k_{1}}{\omega} A u_{0}+\frac{k_{2}}{\omega} B u_{0}=0 .
$$

We have the following theorem :

Theorem 1 There exists a unique plane wave, $v$, solution of system (15) on the form

$$
v\left(x_{1}, x_{2}, t\right)=u_{0} e^{-i\left(k_{1} x_{1}+k_{2} x_{2}-\omega t\right)} e^{\alpha\left(x_{1}\right)},
$$

satisfying :

- $v \equiv u$ in the left half-space $x_{1} \leq 0$ (no reflexion)

- $v$ is damped in the right half-space

- the damping coefficient in the absorbing layer is

$$
\frac{\left|v\left(x_{1}, x_{2}, t\right)\right|}{\left|u\left(x_{1}, x_{2}, t\right)\right|}=e^{\alpha\left(x_{1}\right)}=\exp \left(-\frac{k_{1}}{\omega} \int_{0}^{x_{1}} d(\xi) d \xi\right), \quad x_{1}>0
$$

Proof: See[13]

Remark 5 Note that the damping is exponentially decreasing, depending on the direction of propagation of the wave: it decreases very fast for a wave propagating normally to the interface and more and more slower as the direction approaches the parallel to the interface.

In practice, we introduce a boundary at $x_{1}=\delta$ to bound the layer, with a Dirichlet condition and we solve (13)-(a) in the left half-space and (15) in the right half-space. This new boundary produces a reflexion, but, since the wave decreases exponentially in the layer, the reflexion coefficient becomes quickly very small. This coefficient depends on the choice of $d\left(x_{1}\right)$ and on the size $\delta$ of the layer. One has interest to choose a large enough layer in order to get a small reflexion coefficient, but not too large in order to avoid to increase too much the additional computational cost. 


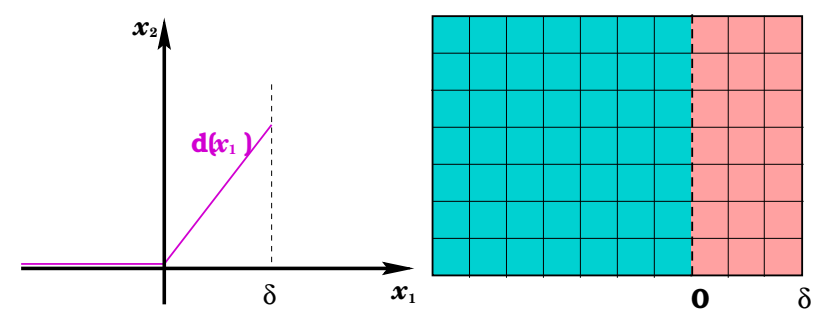

Figure 3: The damping parameter

\subsection{Application to elastodynamics}

Now consider the 2D elastodynamic problem written as a first order hyperbolic system, the velocity-stress system :

$$
\left\{\begin{array}{l}
\varrho \frac{\partial v}{\partial t}-\operatorname{div} \sigma=0 \text { in } \Omega \\
A \frac{\partial \sigma}{\partial t}-\varepsilon(v)=0 \text { in } \Omega
\end{array}\right.
$$

We use the usual identification of tensor $\sigma$ with the vector (still denoted by $\sigma$ ) defined as

$$
\sigma_{1}=\sigma_{11} ; \sigma_{2}=\sigma_{22} ; \sigma_{3}=\sigma_{12}
$$

We can write (19) in the following matricial form :

$$
\begin{cases}\varrho \frac{\partial v}{\partial t}=D^{\perp} \frac{\partial \sigma}{\partial x_{1}}+D^{\|} \frac{\partial \sigma}{\partial x_{2}} & \text { in } \Omega \\ A \frac{\partial \sigma}{\partial t}=E^{\perp} \frac{\partial v}{\partial x_{1}}+E^{\|} \frac{\partial v}{\partial x_{2}} & \text { in } \Omega\end{cases}
$$

with

$$
D^{\|}=\left[\begin{array}{ccc}
0 & 0 & 1 \\
0 & 1 & 0
\end{array}\right] ; D^{\perp}=\left[\begin{array}{ccc}
1 & 0 & 0 \\
0 & 0 & 1
\end{array}\right] ; E^{\|}=\left[\begin{array}{cc}
0 & 0 \\
0 & 1 \\
1 & 0
\end{array}\right] ; E^{\perp}=\left[\begin{array}{cc}
1 & 0 \\
0 & 0 \\
0 & 1
\end{array}\right]
$$

Applying the previous result, we get the following system in the Perfectly Matched Layer

$$
\begin{cases}v=v^{\|}+v^{\perp}, & \sigma=\sigma^{\|}+\sigma^{\perp}, \\ \varrho \frac{\partial v^{\|}}{\partial t}=D^{\|} \frac{\partial \sigma}{\partial x_{2}}, & \varrho \frac{\partial v^{\perp}}{\partial t}+d\left(x_{1}\right) v^{\perp}=D^{\perp} \frac{\partial \sigma}{\partial x_{1}} \\ A \frac{\partial \sigma^{\|}}{\partial t}=E^{\|} \frac{\partial v}{\partial x_{2}}, & A \frac{\partial \sigma^{\perp}}{\partial t}+d\left(x_{1}\right) A \sigma^{\perp}=E^{\perp} \frac{\partial v}{\partial x_{1}} .\end{cases}
$$

\section{Construction of the $Q_{1}-Q_{0}$ mixed finite element}

As explained previously (see section 3.2), in order to achieve mass lumping on matrix $A_{h}$ we have to find appropriate finite element spaces. As this is independent of the presence or not of the boundary $\Gamma_{S}$, we consider the problem without "obstacle", i.e. problem (4), and we assume that $\Omega$ is the square $] 0,1[\times] 0,1[$. We denote by $\mathcal{T}_{h}$ a regular mesh of $\Omega$ composed by squares $(K)$ of side $h=1 / N$. Our aim is to use a space discretisation method that can lead after time discretisation to an explicit scheme. To do so, we are led to construct a new finite element method which fits our aim. For simplicity we present the lowest order element, so-called $Q_{1}-Q_{0}$ 
element, but this construction can be easily generalized to higher orders and to the 3D problem $[8,9]$. We consider the approximate spaces:

$$
\begin{aligned}
& M_{h}=\left\{u_{h} \in M / \forall K \in \mathcal{T}_{h},\left.u_{h}\right|_{K} \in\left(Q_{0}\right)^{2}\right\}, \\
& X_{h}=\left\{\sigma_{h} \in X / \forall K \in \mathcal{T}_{h},\left.\sigma_{h}\right|_{K} \in\left(Q_{1}\right)^{2}\right\}, \\
& X_{h}^{s}=\left\{\sigma_{h} \in X_{h} / \mathbf{a s}\left(\sigma_{h}\right)=0\right\} .
\end{aligned}
$$

In this case the approximation problem, associated to the mixed velocity-stress system for elastodynamics, can be written in the following form :

$$
\left\{\begin{array}{l}
\text { Find }\left(\sigma_{h}, v_{h}\right): \mathbb{R}^{+} \rightarrow X_{h}^{s} \times M_{h} \text { such that : } \\
\frac{d}{d t} a\left(\sigma_{h}(t), \tau_{h}\right)+b\left(\tau_{h}, v_{h}(t)\right)=0, \forall \tau_{h} \in X_{h}^{s}, \\
\frac{d}{d t} c\left(v_{h}(t), w_{h}\right)-b\left(\sigma_{h}(t), w_{h}\right)=\left(f, w_{h}\right), \forall w_{h} \in M_{h},
\end{array}\right.
$$

\section{Remark 6}

- One could think that the most natural choice for the construction of the space $X_{h}$ would be the lowest order Raviart Thomas element[21, 11] $R T_{[0]}$ :

$$
\begin{aligned}
& X_{h}^{R T}=\left\{\sigma_{h} \in X /\left(\sigma_{h 1}, \sigma_{h 2}\right) \in\left(R T_{[0]}\right)^{2}\right\}, \\
& R T_{[0]}=P_{1,0} \times P_{0,1} .
\end{aligned}
$$

The difficulty is that the space $X_{h}^{R T} \cap X^{s}$ is too small and thus it cannot be considered as a good approximation space of $X^{s}:$ if $\sigma_{h}$ is a symmetric tensor in $X_{h}^{R T}$, then $\sigma_{12}$ is necessarily constant! ( $\sigma_{12}$ is linear in $x_{2}$ and constant in $x_{1}$ while $\sigma_{21}$ is linear in $x_{1}$ and constant in $x_{2}$ ).

- Another approach, which was developed for the stationary problem, and permits to avoid this difficulty consists in imposing the symmetry of the stress tensor as $(\sigma)=0$ in a weak way and introducing the corresponding Lagrange multiplier. Following this approach for the transitory problem, we can show that the continuous problem (3) is equivalent to :

$$
\begin{cases}\text { Find }(\sigma, v, \gamma): \mathbb{R}^{+} \rightarrow X \times M \times L \text { such that : } & \\ \frac{d}{d t} a(\sigma(t), \tau)+b(\tau, v(t))+d(\tau, \gamma(t))=0 & \forall \tau \in X^{s} \\ \frac{d}{d t} c(v(t), w)-b(\sigma(t), w)=(f, w) & \forall w \in M \\ d(\sigma(t), \mu)=0 & \forall \mu \in L\end{cases}
$$

where $\gamma$ corresponds to $\operatorname{rot}(v) / 2, L=L^{2}(\Omega)$ and the bilinear form $d(\cdot, \cdot)$ is defined by

$$
d(\sigma, \mu)=\int_{\Omega} \mathbf{a s}(\sigma) \mu d x, \quad \forall(\sigma, \mu) \in H \times M .
$$

It is from this type of formulation, or more precisely from its equivalent for the stationary problem, that PEERS element was constructed[1]. We did not follow this technique because it does not lead to an explicit scheme after time discretisation.

- Our space $X_{h}^{s}$ is constructed from the second family of mixed finite elements proposed by Nédélec[19]. It presents two advantages, the first one concerns the symmetry of the stress tensor which is taken into account in a strong way (that means the symmetry is included in the approximation space), the second one concerns the obtention of an explicit time discretisation scheme using mass-lumping techniques.

Strong symmetry: Consider $\sigma_{h} \in X_{h}^{s}$, we can remark, using the fact that the mesh is regular, that relation $\sigma_{12}=\sigma_{21}$ implies that $\sigma_{12}$ is continuous in $\Omega$ (namely it belongs in the approximation space of $H^{1}$ by $Q_{1}$ finite elements), moreover the vector $\left(\sigma_{11}, \sigma_{22}\right)^{t}$ is now in $H(\operatorname{div}): \sigma_{11}$ is continuous in the $x_{1}$ direction and discontinuous in $x_{2}$ and $\sigma_{22}$ is continuous in $x_{2}$ and discontinuous in $x_{1}$. We can easily show that these properties are consequences of the strong symmetry condition.

Mass lumping: In order to obtain an explicit time discretisation scheme we need to use a mass lumping technique for the approximation of the mass matrix associated to the bilinear form $a\left(\sigma_{h}, \tau_{h}\right)$ (the reader can 
verify that the matrix associated to $c\left(u_{h}, v_{h}\right)$ is already diagonal in the usual basis of $\left.M_{h}\right)$. We first remind that the basic principle of mass-lumping consists in approximating the integrals appearing in the mass matrix using a quadrature formula of the form:

$$
\int_{K} f d x \approx I_{K}(f) \equiv \sum_{i \in K} f\left(M_{i}\right) \omega_{i}
$$

where $M_{i}$ are the quadrature points and $\omega_{i}$ the associated weights. The key point is then to find an adequate quadrature formula which will lead to a diagonal mass matrix. For instance, if we wanted to approximate the scalar acoustic wave equation with the classical $Q_{1}$ element, the integrals appearing in the mass matrix would be $\int_{K} w_{i} w_{j} d x$ with $w_{i}\left(M_{j}\right)=\delta_{i j}$ the Lagrange basis functions and $M_{j}$ the nodes of the mesh (summits of the elements). One can easily check that the use of the following quadrature formula:

$$
\int_{k} f d x \approx I_{K}(f)=\frac{h^{2}}{4} \sum_{M \text { summits of } K} f(M) \quad \forall f \in C^{o}(K)
$$

leads now to a diagonal mass matrix. In our case, following the same principle, we are led to approximate the mass matrix $a\left(\sigma_{h}, \tau_{h}\right)$ by :

$$
a_{h}\left(\sigma_{h}, \tau_{h}\right)=\sum_{K \in \mathcal{T}_{h}} I_{K}\left(A \sigma_{h}: \tau_{h}\right)
$$

where the use of the quadrature formula (24) on $K$ leads now to a block diagonal mass matrix. Each block is associated to a node of the mesh and its dimension is equal to the number of degrees of freedom at this point (that is 5, see figure 4-right).

We present in figure 4 the degrees of freedom in the new element. In figure 4-right we consider a node of
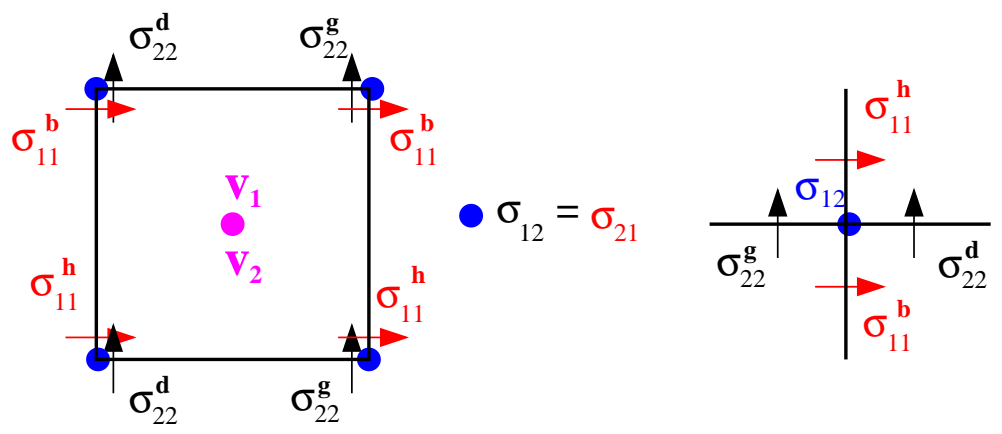

Figure 4: Degrees of freedom for the $Q_{1}-Q_{0}$ element.

the mesh and we present the degrees of freedom (corresponding to the stress tensor) that are associated to this node.

Remark 7 In a recent paper[8], the scalar version of this element is presented, for the approximation of the scalar anisotropic wave equation. In particular, it is explained why the use of Raviart-Thomas elements do not allow to achieve mass-lumping while this element does. For the analysis of this element, the difficulty is that it does not enter the classical Babushka-Brezzi theory (compared to the choice $R T_{[0]}, Q_{0}$ usually used for the pressure and velocity, we have enriched the space of the pressures). That is why we have proposed a modified abstract theory which permits to get nonclassical error estimates. However, the theory developed there can not be directly applied to the elastodynamic problem, it has to be again modified, see [9].

\section{Stability and dispersion analysis of the $Q_{1}-Q_{0}$ element in the case of a homogeneous, isotropic medium.}

In this paragraph we will study the new lowest order mixed finite element method in the case of an homogeneous, isotropic medium. In this case the discretisation scheme obtained using the new finite element, can be interpreted as a finite differences scheme and thus can be analyzed by the usual techniques: dispersion and stability analysis. RR $\quad n^{\circ} 3889$ 


\subsection{Interpretation as a finite difference scheme}

We will describe here the numerical scheme obtained after space discretisation of the problem (23) in a regular mesh. We can see that we have a periodicity of two types of nodes (cf. Fig 5-left). The points 1 and 2 will be
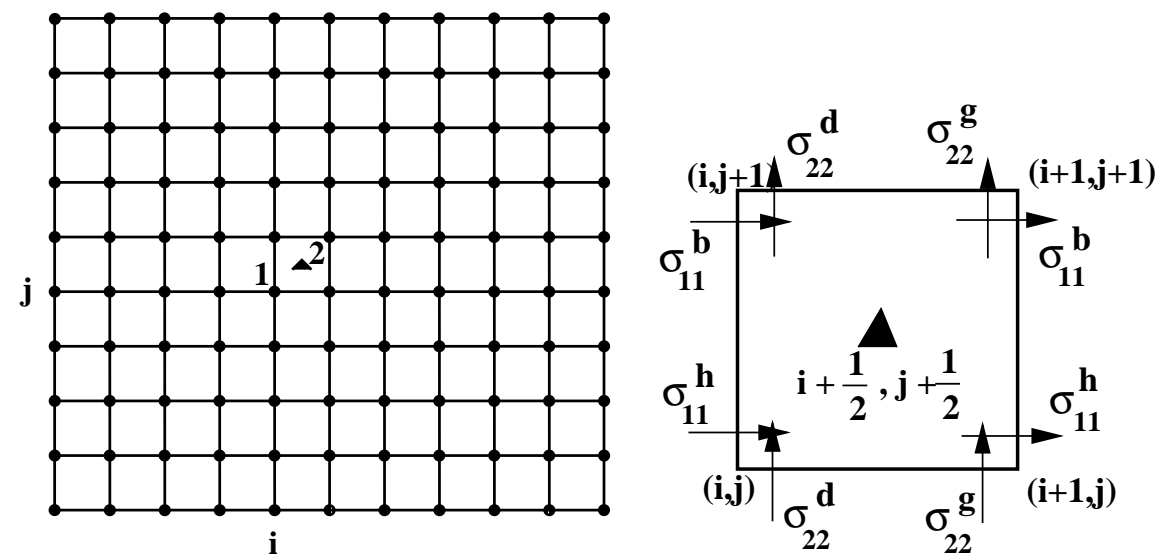

Figure 5: Left: The mesh. Right: Degrees of freedom

respectively indexed by $(i, j)$ and $(i+1 / 2, j+1 / 2)$. At each point of type 1 corresponds 5 degrees of freedom, see Fig. 5-right : $\sigma_{11}^{h}, \sigma_{11}^{b}, \sigma_{22}^{d}, \sigma_{22}^{g}$ and $\sigma_{12}$. At each point of type 2 corresponds 2 degrees of freedom, the two components of the velocity: $v_{1}$ and $v_{2}$. After some calculations (we do not enter here into details), System (23) can be rewritten as a finite difference scheme, and the unknown $\sigma$ can be eliminated. We end up with a second order in time scheme on the velocity, that can be written in a matricial form:

$$
\frac{d^{2} V}{d t^{2}}=\mathbb{K}_{h} V \quad \text { with } \quad \mathbb{K}_{h}=\left[\begin{array}{cc}
V_{p}^{2} \mathcal{D}_{\alpha, 1}^{2}+V_{s}^{2} \mathcal{D}_{\beta, 2}^{2} & \left(V_{p}^{2}-V_{s}^{2}\right) D_{12} \\
\left(V_{p}^{2}-V_{s}^{2}\right) D_{12} & V_{p}^{2} \mathcal{D}_{\alpha, 2}^{2}+V_{s}^{2} \mathcal{D}_{\beta, 1}^{2}
\end{array}\right]
$$

where $V=\left(v_{1}, v_{2}\right)^{t}$ and $\mathcal{D}_{\alpha, 1}^{2} f(i, j)=\alpha D_{1}^{2} f(i, j-1)+(1-2 \alpha) D_{1}^{2} f(i, j)+\alpha D_{1}^{2} f(i, j+1)$, $D_{1}^{2}$ being the discrete operator (classical second order, centered, finite differences operator):

$$
D_{1}^{2} f(i, j)=\frac{f(i+1, j)-2 f(i, j)+f(i-1, j)}{h^{2}}
$$

We can remark that (25) defines a general class of second order numerical schemes, depending on two parameters $\alpha$ and $\beta$ with $(0 \leq \alpha \leq 1 / 2,0 \leq \beta \leq 1 / 2)$. In particular, $\alpha=0, \beta=0$ corresponds to the finite differences scheme, $\alpha=1 / 6, \beta=1 / 6$ to the $Q_{1}$ finite elements scheme and $\alpha=\frac{\left(V_{p}^{2}-2 V_{s}^{2}\right)^{2}}{4 V_{p}^{4}}, \beta=\frac{1}{4}$ to the $Q_{1}-Q_{0}$ mixed finite element scheme. Note that, for the new scheme, $\alpha$ depends on the Poisson's coefficient $\nu=\frac{\lambda}{2(\lambda+\mu)}$, which means that it is adapted to the considered elastic medium.

Remark 8 System (25) is an approximation of the elastodynamic problem, written in displacement:

$$
\frac{d^{2} V}{d t^{2}}=\mathbb{K} V \quad \text { with } \quad \mathbb{K}=\left[\begin{array}{cc}
V_{p}^{2} \frac{\partial^{2}}{\partial x_{1}^{2}}+V_{s}^{2} \frac{\partial^{2}}{\partial x_{2}^{2}} & \left(V_{p}^{2}-V_{s}^{2}\right) \frac{\partial^{2}}{\partial x_{1} \partial x_{2}} \\
\left(V_{p}^{2}-V_{s}^{2}\right) \frac{\partial^{2}}{\partial x_{1} \partial x_{2}} & V_{p}^{2} \frac{\partial^{2}}{\partial x_{2}^{2}}+V_{s}^{2} \frac{\partial^{2}}{\partial x_{1}^{2}}
\end{array}\right]
$$

\subsection{Stability condition}

For the time discretisation of problem (25) we use the classical second order centered finite differences approximation and get the total discretized scheme:

$$
\frac{V^{n+1}-2 V^{n}+V^{n-1}}{\Delta t^{2}}=\mathbb{K}_{h} V^{n}
$$


The stability condition for this scheme is:

$$
\frac{\Delta t^{2}\left\|\mathbb{K}_{h}\right\|}{4} \leq 1
$$

In order to calculate $\left\|\mathbb{K}_{h}\right\|$ we will use the Fourier transform in space :

$$
\forall f\left(x_{1}, x_{2}\right) \in L^{1}\left(\mathbb{R}^{2}\right) \text { we have }: \hat{f}\left(k_{1}, k_{2}\right)=\frac{1}{\sqrt{2 \pi}} \int_{\mathbb{R}^{2}} f\left(x_{1}, x_{2}\right) \exp ^{-i\left(k_{1} x_{1}+k_{2} x_{2}\right)} d x_{1} d x_{2}
$$

From Parseval identity, we get

$$
\left\|\mathbb{K}_{h}\right\|=\sup _{\vec{k}}\left\|\widehat{\mathbb{K}_{h}}(\vec{k})\right\|=\max _{i=1,2} \sup _{\left(X_{1}, X_{2}\right) \in[0,1]^{2}} \lambda_{i}\left(X_{1}, X_{2}\right)
$$

where $\lambda_{i}\left(X_{1}, X_{2}\right), i=1,2$ are the two eigenvalues of the symmetric matrix $\widehat{\mathbb{K}_{h}}$, whose components are expressed in terms of $X_{1}=\sin ^{2}\left(\frac{k_{1} h}{2}\right)$ and $X_{2}=\sin ^{2}\left(\frac{k_{2} h}{2}\right)$ and are:

$$
\left\{\begin{array}{l}
\widehat{\mathbb{K}_{h}}[1,1]=\frac{1}{h^{2}}\left(4 V_{p}^{2} X_{1}\left(1-4 \alpha X_{2}\right)+4 V_{s}^{2} X_{2}\left(1-4 \beta X_{1}\right)\right) \\
\widehat{\mathbb{K}_{h}}[2,2]=\frac{1}{h^{2}}\left(4 V_{p}^{2} X_{2}\left(1-4 \alpha X_{1}\right)+4 V_{s}^{2} X_{1}\left(1-4 \beta X_{2}\right)\right) \\
\widehat{\mathbb{K}_{h}}[1,2]=\frac{4}{h^{2}}\left(V_{p}^{2}-V_{s}^{2}\right) \sqrt{X_{1}\left(1-X_{2}\right)} \sqrt{X_{2}\left(1-X_{1}\right)}
\end{array}\right.
$$

Theorem 2 The scheme (27) is stable under the CFL condition

$$
\frac{V_{C F L} \Delta t}{h} \leq 1, \text { with } V_{C F L}=\frac{h}{2}\left(\max _{i=1,2} \max _{\left(X_{1}, X_{2}\right) \in[0,1]^{2}} \lambda_{i}\left(X_{1}, X_{2}\right)\right)^{1 / 2}
$$

The value of $V_{C F L}$ depends on parameters $\alpha$ and $\beta$, see Fig 6:

- In domain $I: V_{C F L}=\sqrt{V_{p}^{2}+V_{s}^{2}-4\left(\alpha V_{p}^{2}+\beta V_{s}^{2}\right)}$.

- In domain II, the value is constant: $V_{C F L}=V_{p}$.

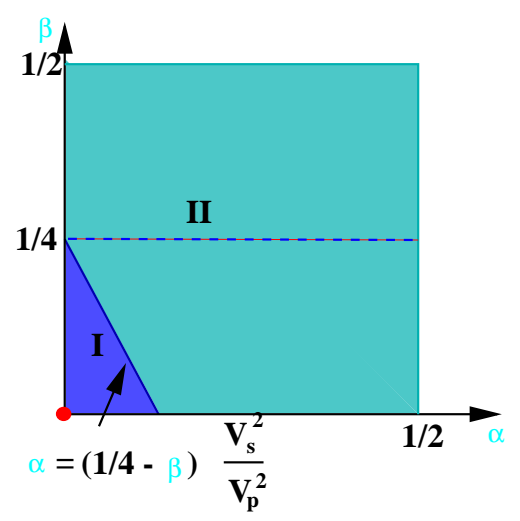

Figure 6: Stability Domains

Proof: We only indicate the main steps of the proof (for more details, see[22]).

- We first study the characteristic polynomial and show that, if the maximum of the greater eigenvalue is reached in the interior of the square, then it is on a point located on the diagonal $X_{1}=X_{2}$. In other terms, this maximum is reached either on the diagonal $X_{1}=X_{2}$ or on the border of the square $[0,1]^{2}$.

- The expressions of the eigenvalues are the following, for $i=1,2$

$$
\begin{aligned}
\lambda_{i}\left(X_{1}, X_{2}\right)= & =\frac{2}{h^{2}}\left[\left(V_{p}^{2}+\beta V_{s}^{2}\right) X_{1} X_{2}\right] \\
& \pm \frac{2}{h^{2}}\left(V_{p}^{2}-V_{s}^{2}\right) \sqrt{\left(X_{1}-X_{2}\right)^{2}+4\left(X_{1}-X_{1}^{2}\right)\left(X_{2}-X_{2}^{2}\right)}
\end{aligned}
$$

$\mathrm{RR} \mathrm{n}^{\circ} 3889$ 
We then prove that the maximum of the greater one is necessarily reached at one vertex of the square and get the result.

For the finite differences scheme $(\alpha=\beta=0)$ the maximum is in domain I, $V_{C F L}^{F D}=\sqrt{V_{p}^{2}+V_{s}^{2}}$, while for the $Q_{1}$ finite elements as well as for the $Q_{1}-Q_{0}$ mixed finite element the maximum is in domain II, $V_{C F L}^{Q_{1}}=V_{C F L}^{Q_{1}-Q_{0}}=V_{p}<V_{C F L}^{F D}$, and therefore is better than with finite differences. In the following, we will call CFL ratio the quantity $\alpha_{C F L}=\Delta t / h$ and we denote by $\alpha_{C F L}^{F D}, \alpha_{C F L}^{Q_{1}}=\alpha_{C F L}^{Q_{1}-Q_{0}}$ the maximum allowed by the stability condition for each scheme (i.e., $\alpha_{C F L}^{F D}=1 / V_{C F L}^{F D}<\alpha_{C F L}^{Q_{1}}=1 / V_{C F L}^{Q_{1}}$ ).

\subsection{Dispersion Analysis for the total discretized scheme}

For the continuous problem, the dispersion analysis consists in searching plane waves solutions of system (26), i.e. waves in the following form:

$$
U=U_{o} \exp \imath\left(k_{1} x_{1}+k_{2} x_{2}-\omega t\right), \quad U_{o} \in \mathbb{R}^{2}, \quad k=\left(k_{1}, k_{2}\right) \in \mathbb{R}^{2}, \omega \in \mathbb{R}
$$

where $k$ is the propagating direction and $\omega / k$ the phase velocity. To be a solution, $U$ has to verify the so called dispersion relation :

$$
\omega^{2} U_{o}=\widehat{\mathbb{K}} U_{o} \quad \text { where } \quad \widehat{\mathbb{K}}=\left[\begin{array}{cc}
V_{p}^{2} k_{1}^{2}+V_{s}^{2} k_{2}^{2} & \left(V_{p}^{2}-V_{s}^{2}\right) k_{1} k_{2} \\
\left(V_{p}^{2}-V_{s}^{2}\right) k_{1} k_{2} & V_{p}^{2} k_{2}^{2}+V_{s}^{2} k_{1}^{2}
\end{array}\right]
$$

Equation (33) implies that $\omega^{2}$ is an eigenvalue of $\widehat{\mathbb{K}}$ and $U_{o}$ is the associated eigenvector. Therefore, we have[4]

$$
\begin{array}{ll}
\omega_{1}^{2}=V_{p}^{2}\left(k_{1}^{2}+k_{2}^{2}\right), & U_{o}^{1}=\left(k_{1}, k_{2}\right) \\
\omega_{2}^{2}=V_{s}^{2}\left(k_{1}^{2}+k_{2}^{2}\right), & U_{o}^{2}=\left(-k_{2}, k_{1}\right)
\end{array}
$$

where $\omega_{1}$ corresponds to a Pressure wave, propagating with the phase velocity $V_{p}=\omega_{1} /|k|$ and $\omega_{2}$ corresponds to a Shear wave, propagating with the phase velocity $V_{s}=\omega_{2} /|k|$. Phase velocities $V_{p}$ and $V_{s}$ are independent on $\omega$, we say that the elastodynamic equation is non-dispersive. To study the dispersion relation of scheme (27) we search particular solutions of (25) in the form:

$$
U_{i j}=U_{o h} \exp \imath\left(k_{1} x_{1}^{i}+k_{2} x_{2}^{j}-\omega t\right), \quad U_{o h} \in \mathbb{R}^{2}, \quad k=\left(k_{1}, k_{2}\right) \in \mathbb{R}^{2}, \omega \in \mathbb{R}
$$

We can prove the existence of numerical waves $\mathrm{P}$ and $\mathrm{S}$ which are dispersive. The dispersion relation for scheme (27) is:

$$
\frac{4}{\Delta t^{2}} \sin ^{2}\left(\frac{\Delta t \omega_{h}}{2}\right) U_{o h}=\widehat{\mathbb{K}_{h}} U_{o h}
$$

where $\widehat{\mathbb{K}_{h}}$ is the symmetric matrix defined by (29). We then obtain for $i=1,2$

$$
\frac{4}{\Delta t^{2}} \sin ^{2}\left(\frac{\Delta t \omega_{h}^{i}}{2}\right)=\lambda_{i}\left(X_{1}, X_{2}\right), \quad\left(\lambda_{i} \text { defined in }(31)\right)
$$

We set $k=\left(k_{1}, k_{2}\right)$ where the components of $k$ are related to the angle of propagation $\phi$ by $k_{1}=|k| \cos \phi, \quad k_{2}=$ $|k| \sin \phi$. Let $V_{h}=\frac{\omega_{h}}{|k|}$ the numerical phase velocity. We introduce the adimensional quantities $q_{p}$ (resp. $q_{s}$ ) which represents the ratio between the numerical and the continuous phase velocity of the $\mathrm{P}$ (resp. S) waves :

$$
q_{p}=\frac{\omega_{h}^{1}}{|k| V_{p}} ; q_{s}=\frac{\omega_{h}^{2}}{|k| V_{s}}
$$

If we set $K=\frac{1}{N}=\frac{|k| h}{2 \pi}$, with $N$ the number of points per wavelength, we notice that $q_{p}$ (resp. $q_{s}$ ) depends on the discretisation parameter $K$, on the angle of propagation $\phi$, on the Poisson's coefficient $\nu=\frac{\lambda}{2(\lambda+\mu)}$ and on the step size $\Delta t$ (or equivalently on the CFL ratio $\alpha_{C F L}$ ). We compare the dispersion of the $Q_{1}-Q_{0}$ mixed 

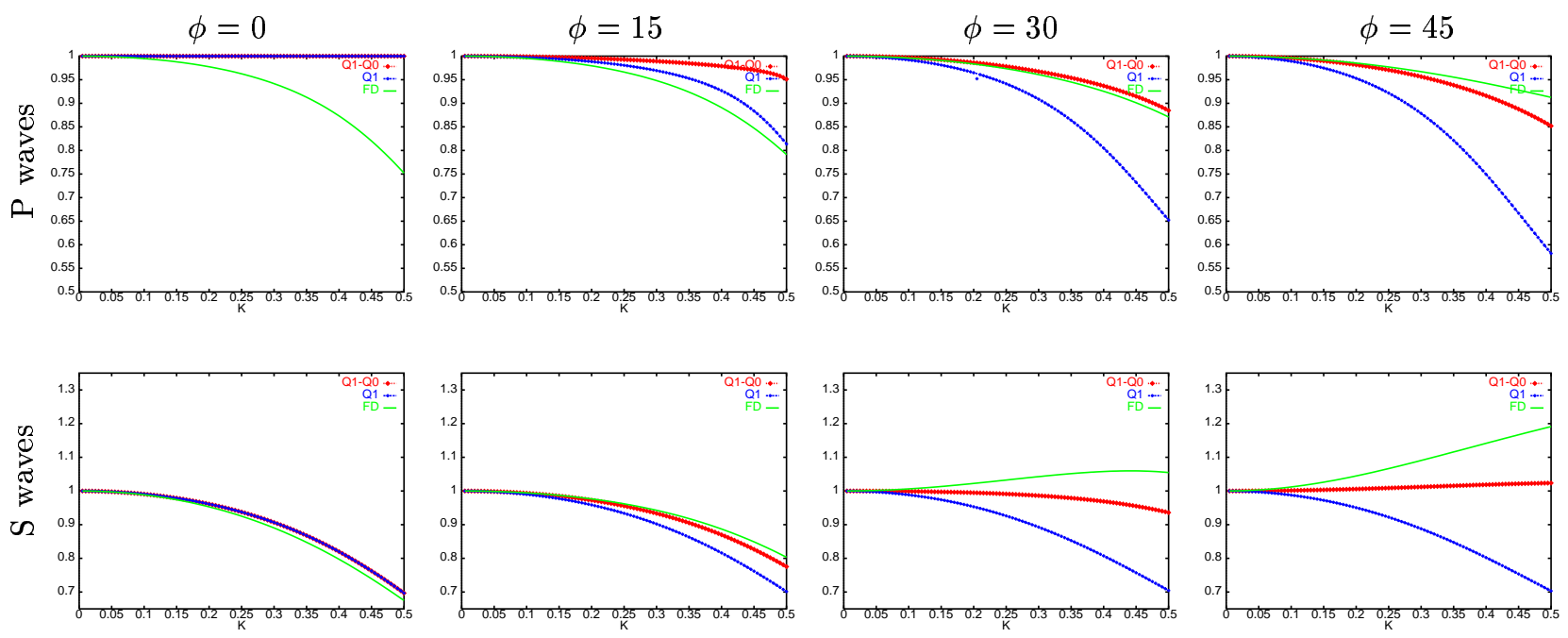

Figure 7: Phase velocity, $\nu=0.1$.
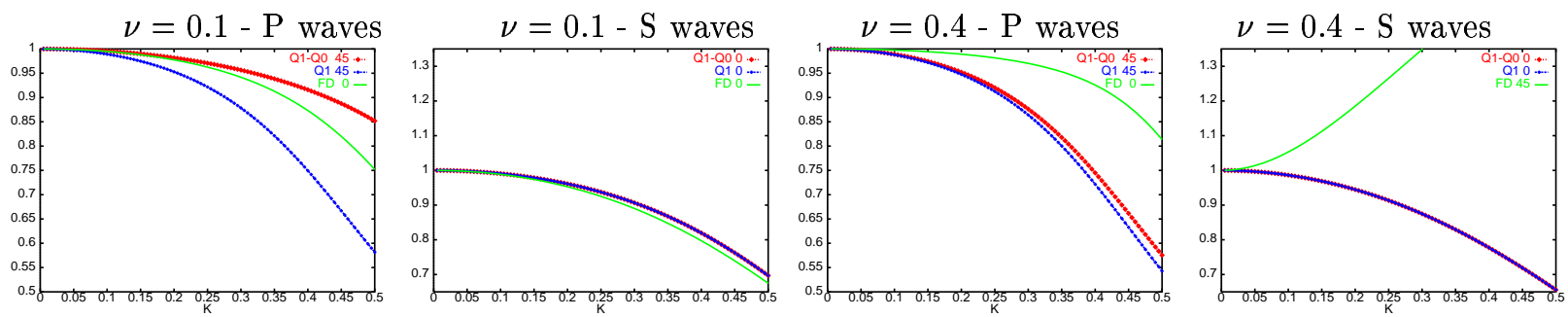

Figure 8: Comparison between the worse curves for $\nu=0.1$ and $\nu=0.4$

finite element scheme (red), the dispersion of the classical $Q_{1}$ finite element scheme (blue) and the dispersion of the classical finite difference scheme (green). For each scheme, we plot $q_{p}$ and $q_{s}$ with respect to $K$, and this for several values of the Poisson's coefficient, for several angles and for the maximum value of the CFL ratio $\alpha_{C F L}$ allowed by the stability condition. Actually, we can check that the dispersion error for $\mathrm{P}$ waves decreases as $\alpha_{C F L}$ increases, we have thus chosen to represent the best curves for the dispersion of $\mathrm{P}$ waves. We present in Fig. 7 the curves obtained for $\nu=0.1$, and for the angles $\phi=0,15,30$ and 45 degrees, but the observations we do here are the same for other values of $\nu$. Concerning $\mathrm{P}$ waves: the $Q_{1}-Q_{0}$ and $Q_{1}$ elements have their worse dispersion for the diagonal direction $\phi=45$ and become better when the direction becomes parallel to the grid axis $\phi=0$, and it is the contrary for the FD scheme. For $\mathrm{S}$ waves, the $Q_{1}-Q_{0}$ and $Q_{1}$ elements have again a monotone behavior with respect to the angle, which is inverted compared to $\mathrm{P}$ waves, i.e., they have their worse dispersion for $\phi=0$ and become better for $\phi=45$. On the other hand, one can not conclude for the FD scheme, since the results change depending on the angle and on the value of $\nu$.

We represent in Fig 8 the "worse" curves for each scheme, for two values of $\nu$. We can see that, in all cases, the new finite element $Q_{1}-Q_{0}$ is always better (or identical) than the $Q_{1}$ element (with the same CFL ratio). The comparison with FD is not so clear for $\mathrm{P}$ waves: if we fix the number of points per wavelength, the FD can become in some cases better than the $Q_{1}-Q_{0}$ (e.g., $\nu=0.4, \mathrm{P}$ waves) but with a smaller CFL ratio, i.e. it is also more expensive. On the other hand, the $Q_{1}-Q_{0}$ always gives a better (or identical) dispersion than FD for $\mathrm{S}$ waves, which are slower than $\mathrm{P}$ waves $\left(V_{s}<V_{p}\right)$ and thus more difficult to approximate.

In conclusion, if we consider both stability and dispersion, our scheme has a better behavior than Finite Differences and than $Q_{1}$. This was not apriori expected since this element was designed for different purposes (strong symmetry and mass lumping).

\section{Numerical Results}

We will present in this section three numerical experiments. In the two first examples, without topography, we want to validate the lowest order new mixed finite element and the absorbing layers in isotropic and anisotropic 
media. The last example shows the efficiency of the coupling with the fictitious domain method in a heterogeneous medium. For the time discretisation we use the classical centered finite difference scheme of 2 nd order. In each case, we solve the elastodynamics problem with zero initial conditions, and with an explosive source located at point $S=\left(x_{s}, y_{s}\right)$,

$$
f(x, t)=F(t) g(r)
$$

where

$$
\begin{cases}F(t)=\left\{\begin{array}{ll}
-2 \pi^{2} f_{0}^{2}\left(t-t_{0}\right) e^{-\pi^{2} f_{0}^{2}\left(t-t_{0}\right)^{2}} & \text { if } t \leq 2 t_{0} \\
0 & \text { if } t>2 t_{0}
\end{array},\right. \\
t_{0}=\frac{1}{f_{0}}, \quad f_{0}=\frac{V_{s}}{h} \frac{1}{N_{S}} \text { is the central frequency, } \\
N_{S} \text { is the number of points per } \mathrm{S} \text { wave length, }\end{cases}
$$

and $\vec{g}(r)$ is a radial function :

$$
\left\{\begin{array}{l}
\vec{g}(r)=\left(1-\frac{r^{2}}{a^{2}}\right)^{3} 1_{B_{a}} \vec{e} \\
r=\sqrt{\left(x-x^{s}\right)^{2}+\left(y-y^{s}\right)^{2}}, a=5 h,
\end{array}\right.
$$

where $1_{B_{a}}$ is one on $B_{a}$, the disk of center $\mathrm{S}$ and radius $a$, and zero elsewhere and $\vec{e}$ will be precised in each experiment.

The absorbing layer model is characterized by the length $\delta$ of the layer, and the damping parameter (see figure 9-right)

$$
d(x)=d_{0}\left(\frac{x}{\delta}\right)^{2}, \quad \text { with } d_{0}=\left|\log \left(\frac{1}{R}\right)\right| \frac{3 V_{p}}{2 \delta}
$$

and $R$ is the reflection coefficient predicted by the theory [13], $\delta$ and $R$ being defined in each case. The domain $C$ is meshed with $N \times N$ squares of edge $h$.

The time step is computed following the CFL condition $\Delta t=\frac{h}{V_{p}}$.

Remark 9 In a heterogeneous medium, we choose

$$
f_{0}=\frac{\min V_{s}}{h} \frac{1}{N_{S}}, \quad d_{0}=\left|\log \left(\frac{1}{R}\right)\right| \frac{3 \max V_{p}}{2 \delta}, \quad \text { and } \Delta t=\frac{h}{\max V_{p}}
$$

where the $\max$ and the min are the extremal values of the velocities on the whole domain.

\subsection{Rayleigh waves and PML}

In the first example we consider the elastic wave propagation problem in the homogeneous isotropic half plane $(y<0)$ where the source point $S$ is near the free surface. We have chosen this problem in order to test the efficiency of the P.M.L model on the Rayleigh wave, which is particularly difficult to absorb. In order to approximate this problem we consider a bounded domain $C=[0,100] \times[-100,0]$ with absorbing layers (PML) on the artificial boundary $\Gamma_{A}$ (see Figure 9-left). The velocities in the medium are $V_{p}=\sqrt{5}, V_{s}=\sqrt{2}$. The uniform grid on $C$ is composed of squares of edge $h=0.5$. To complete the characteristics of the source, given in (38) and (39), we choose $\vec{e}=\left(\frac{x-x_{s}}{r}, \frac{y-y_{s}}{r}\right)$ and the point source $S=(50,97)$. The length of the absorbing layer is $\delta=10 h$ and the theoretical reflection coefficient is $R=0.001$. In Figure 10, we represent the solution of this problem at different times. We can see two cylindrical waves propagating with two different velocities (Pressure wave and Shear wave) and we can also observe the Rayleigh wave propagating along the surface with a velocity roughly equal to the velocity of shear waves. We can remark that P.M.L model absorbs efficiently the cylindrical waves $\mathrm{P}, \mathrm{S}$ and the Rayleigh wave. More precisely the reflection coefficient is in this case $R=0.001$ : this is the value predicted by the theory, and looking carefully on the figures (change of scale), we can check that this is also approximately the numerical value. 

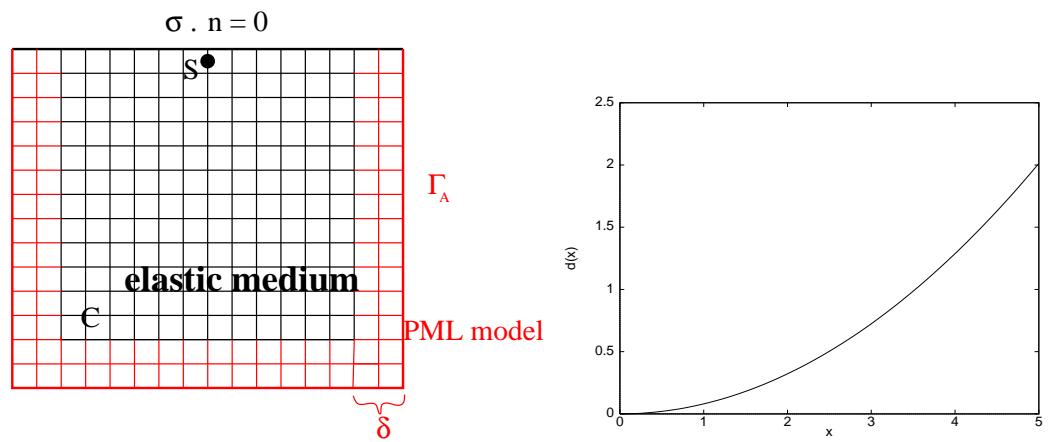

Figure 9: Left: The bounded domain problem. Right:The damping coefficient $d(x)$
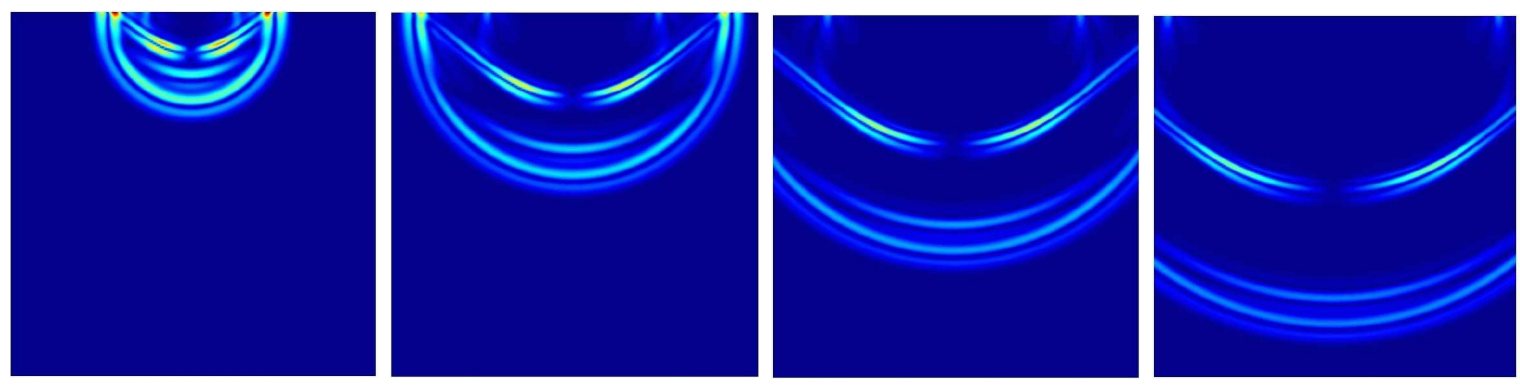

Figure 10: The norm of the velocity $\left(\sqrt{v_{1}^{2}+v_{2}^{2}}\right)$ at time $t=13.44 \mathrm{~s}$ on the left, $t=26.88 \mathrm{~s}$ on the right

\subsection{Homogeneous, anisotropic elastic medium.}

We consider here a homogeneous, anisotropic, elastic medium: the apatite. Again the computational domain is a square $C$ surrounded with absorbing layers on all four boundaries. The characteristics of the problem are : $N=240, h=0.33 m, N_{S}=10$. We use here a z-directional point load source, $\vec{e}=(0,1)$. The source is located at the center of the frame $S=(40 m, 40 m)$. The length of the absorbing layers is $\delta=5 h$ and the reflection coefficient $R=0.01$. The density of the material is $\rho=3.2 \mathrm{gr} / \mathrm{cm}^{3}$ and the matrix of elastic coefficients is

$$
C=\left(\begin{array}{ccc}
16.7 & 6.6 & 0 . \\
6.6 & 14 . & 0 . \\
0 & 0 & 6.63
\end{array}\right) 10^{11}
$$

In a 2D anisotropic medium, there are two waves propagating, the quasi pressure wave (QP) and the quasi shear wave (QS). Before giving the numerical results, we present in Figure 11 the theoretical wave fronts curves for the Apatite and the amplitudes of the QP and QS wave for a a z-directional point load source. In the following
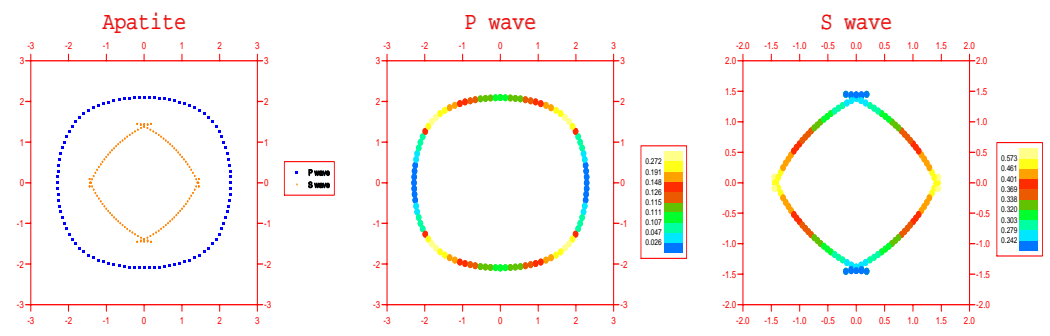

Figure 11: Left: Wave front curves for the apatite.Right: amplitude of the $\mathrm{P}$ wave (left) and $\mathrm{S}$ wave (right)

figures, we represent the solution of this problem at different times.

We can remark that the wave front curves and the amplitude of the Quasi Pressure and the Quasi Shear wave computed numerically show the characteristics predicted theoretically : the Quasi Pressure wave is weaker then the Quasi Shear wave, the amplitude of the Quasi Pressure wave is weaker in the $\mathrm{x}$-direction while the 

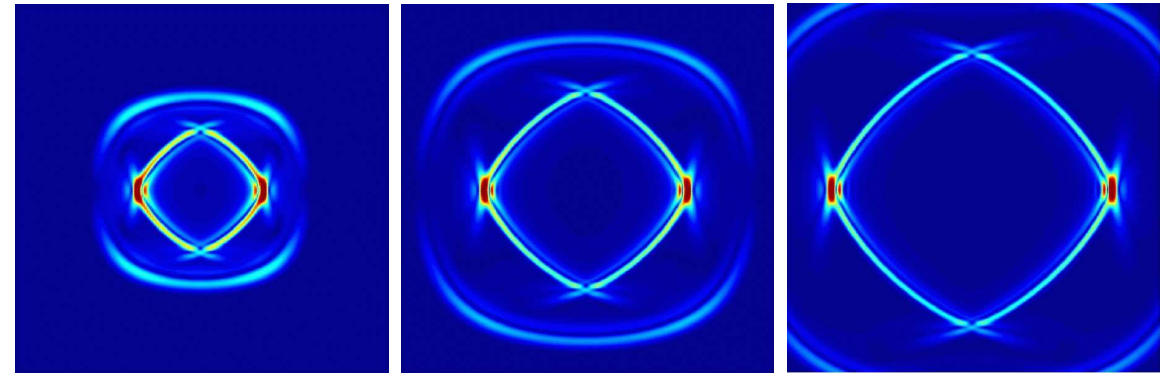

Figure 12: $|\vec{u}|=\sqrt{u_{x}^{2}+u_{y}^{2}}$ at $t=11.67 s, 17.51 s$ and $23.35 s$

amplitude of the Quasi Shear wave is weaker in the y-direction.

\subsection{Coupling with the fictitious domain method: the case of an heterogeneous elastic medium with complex topography}

We consider here, the elastic wave propagation problem in a heterogeneous, isotropic medium with complex topography. To approximate this problem we consider a bounded domain $C=[0,80] \times[0,80]$ with absorbing layers (PML) on the artificial boundary $\Gamma_{A}$ (see Figure 13). As we can see in figure 13 the mesh on the domain $C$ is independent on the mesh on $\Gamma_{S}$. The heterogeneous elastic medium considered here is characterized by
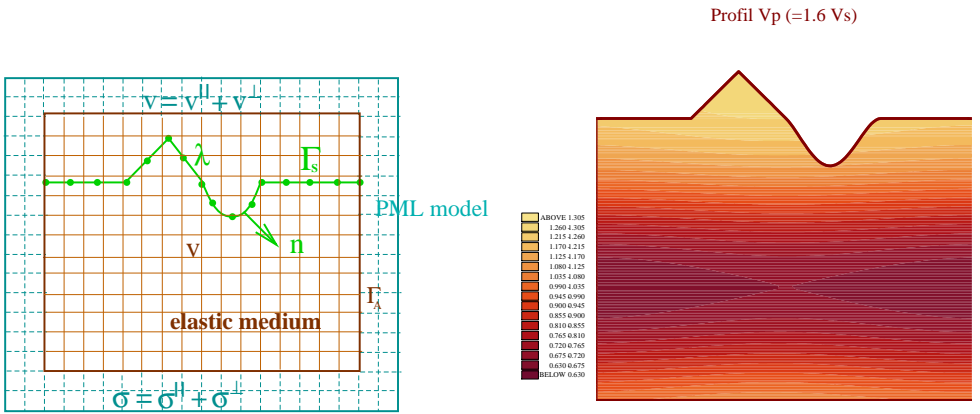

Figure 13: Left: The two meshes. Right: The velocity model for the heterogeneous medium, $\frac{\max V_{p}}{\min V_{p}}=2.1$ and $V_{p}=1.6 V_{s}$.

the velocity model presented on Figure 13-right, we have $\frac{\max V_{p}}{\min V_{p}}=2.1$ and $V_{p}=1.6 V_{s}$. For the discretization we take $V_{p}$ and $V_{s}$ piecewise constants (one value per element). The step of the uniform grid on $C$ is $h=1 / 3$, and the number of points per $\mathrm{S}$ wavelength (for the minimum $\mathrm{S}$ velocity, see remark 9 ) is $N_{S}=10$. The source is determined by $\vec{e}=\left(\frac{x-x_{s}}{r}, \frac{y-y_{s}}{r}\right)$ and the point source is located at $S=(36.67,56.67)$. On the free surface $\Gamma_{S}$ we use an 1D irregular mesh. The length of the absorbing layer is $\delta=10 h$ and the damping parameter is choosen according to (40) and Remark 9 with a reflection coefficient $R=0.01$. In the following figures, we represent the norm of the velocity at different times. We can observe that the wave fronts are not circular, because of the heterogeneities, and the energy is localized in some regions. Although the phenomena are more complicated than in a homogeneous medium, one can see $\mathrm{P}$ and $\mathrm{S}$ waves reflected by the topography (the $\mathrm{P}$ wave being faster than the $\mathrm{S}$ one), and one can also see a diffraction by the wedge. There is a surface wave propagating along the topography, which is again very well absorbed by the PMLs.

\section{Conclusion}

We have presented a new method for solving elastodynamics problem in anisotropic, heterogeneous media, with topographies of complex geometries. The numerical results, obtained with the lowest order element, show its 


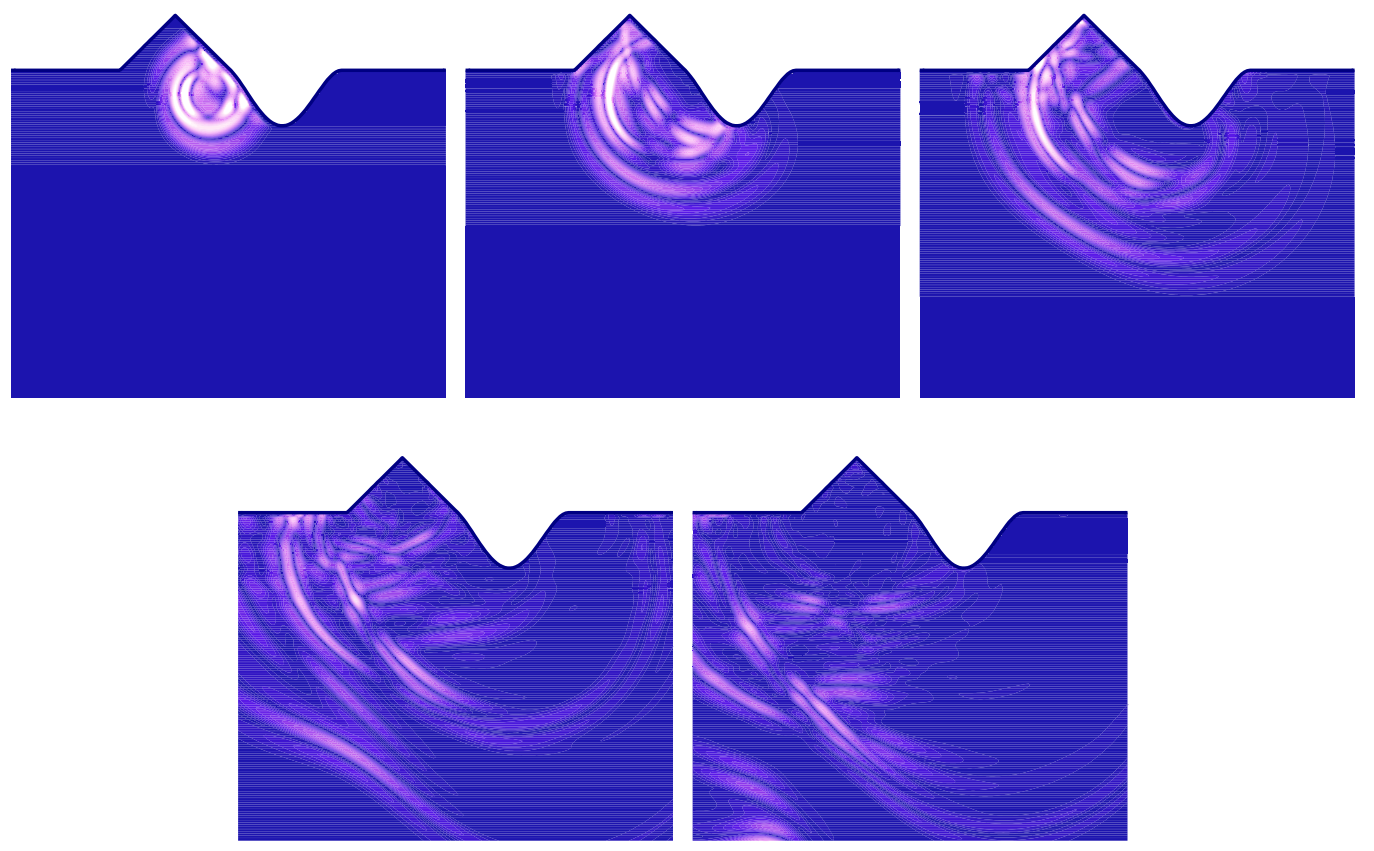

Figure 14: $|\vec{u}|=\sqrt{u_{x}^{2}+u_{y}^{2}}$ at $t=19.35 s, 32.25 s, 45.15 s, 70.95 s$ and $96.75 s$

efficiency in several situations. Let us mention that this method can also be applied in media containing cracks (see[6]). The new elements can be extended in a natural way to higher orders[22] and to 3D. A forthcomming paper is in preparation for the 3D case, where we treat in particular the additional difficulties linked to the interaction between the boundary mesh and the volume mesh, which involves much more geometry than in $2 \mathrm{D}$.

\section{Acknowledgments}

This research was supported by a research contract between EDF/DER and INRIA. This was also a contribution to the Seismic Inversion, Geophysical Modeling and Applications Consortium Project.

\section{References}

[1] N. Arnold, F. Brezzi, and J. Douglas. PEERS: A new mixed finite element for plane elasticity. Japan J. Appl. Math., 1:347-367, 1984.

[2] C. Atamian and P. Joly. Une analyse de la méthode des domaines fictifs pour le problème de Helmholtz extérieur. Technical Report 1378, INRIA, 1991.

[3] B. A. Auld. Acoustic Fields and Elastic Waves in Solids, volume I et II. Wiley, 1973.

[4] A. Bamberger, G. Chavent, and P. Lailly. Étude de schémas numériques pour les équations de l' élastodynamique linéaire. Technical report, INRIA, 1980. $\mathrm{n}^{\circ} 41$.

[5] E. Bécache and T. Ha Duong. A Space-Time Variational Formulation for the Boundary Integral Equation in a 2D Elastic Crack Problem. RAIRO, M2AN, 28,n ${ }^{\circ} 2: 141-176,94$.

[6] E. Bécache, P. Joly, and C. Tsogka. Some numerical experiments - applications of the fictitious domain method to elastodynamic waves. http://www-rocq.inria.fr/ ${ }^{\sim}$ becache/numer.html.

[7] E. Bécache, P. Joly, and C. Tsogka. Eléments finis mixtes et condensation de masse en élastodynamique linéaire. (i) construction. C.R. Acad. Sci. Paris, t. 325, Série I:545-550, 1997.

[8] E. Bécache, P. Joly, and C. Tsogka. Some new mixed finite elements in view of the numerical solution of time dependent wave propagation problems. Technical Report 3445, INRIA, June 1998. submitted in SIAM J. Num. An. 
[9] E. Bécache, P. Joly, and C. Tsogka. A new family of mixed finite elements for the linear elastodynamic problem. Technical report, INRIA, 1999. submitted in SIAM J. Num. An.

[10] J.P. Bérenger. A perfectly matched layer for the absorption of electromagnetic waves. Journal of Comp. Physics., 114:185-200, 1994.

[11] F. Brezzi and M. Fortin. Mixed and Hybrid Finite Element Methods. Springen-Verlag, 1991.

[12] F. Collino, P. Joly, and F. Millot. Fictitious domain method for unsteady problems: Application to electromagnetic scattering. Technical Report 2963, INRIA, Août 1996.

[13] F. Collino and C. Tsogka. Application of the PML Absorbing Layer Model to the Linear Elastodynamic Problem in Anisotropic Heteregeneous Media. Rapport de Recherche 3471, INRIA., 1998. submitted to Geophysics.

[14] M.A. Dablain. The application of high order differencing for the scalar wave equation. Geophysics, 1(51):54$66,1986$.

[15] S. Garcès. Application des méthodes de domaines fictifs à la modélisation des structures rayonnantes tridimensionnelles. $\mathrm{PhD}$ thesis, 1998.

[16] R. Glowinski, T.W. Pan, and J. Periaux. A fictitious domain method for Dirichlet problem and applications. Comp. Meth. in Appl. Mech. and Eng., pages 283-303, 1994.

[17] R. Glowinski, T.W. Pan, and J. Periaux. A fictitious domain method for external incompressible viscous flow modeled by Navier-Stokes equations. Comp. Meth. in Appl. Mech. and Eng., pages 283-303, 1994.

[18] M. E. Morley. A family of mixed finite elements for linear elasticity. Numer. Math., 55:633-666, 1989.

[19] J.C. Nédélec. A new family of mixed finite elements in $\mathbb{R}^{3}$. Numer. Math., 50:57-81, 1986.

[20] R. Stenberg. A family of mixed finite elements for the elasticity problem. Numer. Math., 53:513-538, 1988.

[21] P.A. Raviart and J.M. Thomas. A mixed finite element method for 2nd order elliptic problems. Number 606, pages 292-315. Proc. of math. aspects on the finite element method, 1977.

[22] C. Tsogka. Méthodes de haute précision pour la résolution de l'équation de l'élastodynamique en $3 D$ dans des milieux hétérogènes comportant des fissures. PhD thesis, University Paris IX, 1999. to appear.

[23] V.Girault and R. Glowinski. Error analysis of a fictitious domain method applied to a Dirichlet problem. Japan J. Indust. Appl. Math., 12(3):487-514, 1995. 
Unit'e de recherche INRIA Lorraine, Technopôle de Nancy-Brabois, Campus scientifique, 615 rue du Jardin Botanique, BP 101, 54600 VILLERS LÈS NANCY

Unit'e de recherche INRIA Rennes, Irisa, Campus universitaire de Beaulieu, 35042 RENNES Cedex Unit'e de recherche INRIA Rhône-Alpes, 655, avenue de l'Europe, 38330 MONTBONNOT ST MARTIN Unit'e de recherche INRIA Rocquencourt, Domaine de Voluceau, Rocquencourt, BP 105, 78153 LE CHESNAY Cedex Unit ${ }^{\prime}$ de recherche INRIA Sophia-Antipolis, 2004 route des Lucioles, BP 93, 06902 SOPHIA-ANTIPOLIS Cedex

\section{Éditeur}

INRIA, Domaine de Voluceau, Rocquencourt, BP 105, 78153 LE CHESNAY Cedex (France) 